\title{
Hybrid Optical and Wireless Technology Integrations for Next Generation Broadband Access Networks
}

\author{
N. Moradpoor*, G. Parr, S. McClean and B. Scotney \\ India-UK Advanced Technology Centre of Excellence in \\ Next Generation Networks, Systems and Services \\ School of Computing and Information Engineering \\ University of Ulster, Coleraine, UK, BT52 1SA
}

\author{
G. Owusu \\ BT Laboratories, Adastral Park, UK \\ *E-mail: moradpoor_sheykhkanloo-n@email.ulster.ac.uk \\ Member, IEEE \& OSA
}

\begin{abstract}
Hybrid optical and wireless technology integrations have been considered as one of the most promising candidates for the next generation broadband access networks for quite some time. The integration scheme provides the bandwidth advantages of the optical networks and mobility features of the wireless networks for Subscriber Stations (SSs). It also brings economic efficiency to the network providers particularly in rural area where the existing wired telecommunication infrastructures such as Digital Subscriber Line (DSL), Cable Modem (CM), T-1/E-1 networks or fibre deployments are either costly or unreachable. For successful integration of the optical and wireless technologies there are some technical issues which need to be addressed efficiently in order to provide End-to-End (ETE) and diverse Quality of Service (QoS) for various service classes. This paper investigates the possible challenging issues for the integrated structure of the Time Division Multiplexing and Wavelength Division Multiplexing Ethernet Passive Optical Networks (TDM EPON and WDM EPON ) with the Worldwide Interoperability for Microwave Access and Wireless Fidelity (WiMAX and Wi-Fi) networks. To reduce the ETE delay and provide the QoS for diverse service classes, we have compared six existing upstream scheduling mechanisms in two levels which are distributed on Access Points (APs) from Wi-Fi domain and Base Stations (BSs) from WiMAX domain. Performance evaluations of the existing scheduling techniques for three popular service classes (Quad-play) have been studied which show the strong impact of using the efficient up-link scheduler in converged scenario. We have also proposed a dynamic scheduling algorithm for optical and wireless integration scheme, which is under the implementation and evaluation process.
\end{abstract}

Keywords: TDM EPON, WDM EPON, WiMAX, Wi-Fi, optical and wireless integration

\section{INTRODUCTION}

During the last decade a huge deployment of broadband access to both fixed and mobile telecommunication infrastructures was carried out which requires a mix of the wired and wireless technology generations. The Digital Subscriber Line (DSL) and Cable Modem (CM) networks are the most deployed solutions for the wired broadband access networks. Their problem domains could be regarded as: physical location, upgradability and traffic growth. For instance, they are unable to support subscribers located more than $5.5 \mathrm{~km}$ from the central office (CO). They also cannot provide enough bandwidth for ongoing demands for more sophisticated services and bandwidth hungry applications such as online computer games, music, multimedia, video conferencing, etc. The Worldwide Interoperability for
Microwave Access (WiMAX, IEEE 802.16) is one of the promising candidates among wireless access technologies which can provide low-cost, wide-coverage, fixed and mobile broadband access networks. It can be implemented in two modes: point-to-multi-point (PMP) mode in which there are only communications between the Base Station (BS) and Subscriber Stations (SSs) and mesh mode in which the SSs can also communicate with each other. WiMAX is also capable of providing broadband access for rural area where the existing wired infrastructures such as DSL, CM, T-1/E-1 or even optical cables are either unreachable or too costly. However, WiMAX backhaul technology is still a controversial and challenging issue. The T-1/E-1 and DSL have been proposed for the mobile backhaul technology for quite some time. The $\mathrm{T}-1 / \mathrm{E}-1$ provides diverse $\mathrm{Q} O \mathrm{~S}$ and supports up to $2 \mathrm{Mbps}$ transmitting rate however, it is not a cost-aware and scalable solution. The DSL can also provide high-speed broadband access technology by using the existing telephone lines. However, its performance depends on the subscriber distance from the $\mathrm{CO}$ as well as the time of the access. The Optical Network (ON) is a solution for the DSL and CM problem domains by providing higher bandwidth and supporting subscribers located more than $20 \mathrm{~km}$ from CO. The ON can also be upgraded to higher bit rates or even additional wavelengths easily. The Passive Optical Network (PON) is the most promising candidate among the other ON solutions as it eliminates the necessity of installing the active components such as the multiplexer and de-multiplexer in the splitting point and minimises the fibre deployment between the local exchange and the local loop. Therefore, it is excellent in terms of maintainability and robustness [24]. PON is a point-to-multi-point fibre infrastructure which includes the Optical Line Terminal (OLT) in the CO, the Optical Network Units (ONUs) near the customer premises as well as the $1: \mathrm{N}$ passive splitter/combiner by the split ratio of 16,32 or 64 . The PON can be in the format of the fibre-tothe-home (FTTH), fibre-to-the-PC (FTTPC), fibre-to-thebuilding (FTTB) and fibre-to-the-curb (FTTC) with the ring, bus and tree as the possible network topologies. Ethernet PON (EPON) [5] and Gigabit PON (GPON) [3] are the two major standards for PON [6]. The Ethernet-based Passive Optical Network (EPON, IEEE 802.3ah [5]) appears to be the preferred choice among the other PON's data-link technology such as the SONET-based PON or ATM-based PON[16], e.g. in ATM-PON (APON) the IP packets should be broken into cells in source and then re-assembled at the other end which adds additional complexity and cost to the network. The SONET-based PON is also too expensive for the local loop 
and it is not efficient for the data traffic. On the other hand, the Ethernet is an inexpensive, ubiquitous and scalable (100Mbps, 1Gbps, 10Gbps) technology. It is also interoperable with a variety of legacy equipment with the adopted quality of service (QoS). The possible candidate architectures for the Next Generation PONs have been discussed in [20]. The Time Division Multiplexing (TDM), Wavelength Division Multiplexing (WDM) and the Code Division Multiplexing (CDM) are the possible media-access technologies in PON with different challenging issues in terms of cost and complexity. A comprehensive review of different resource management techniques for TDM PON has been done in [2]. Generally speaking, the PON particularly EPON and WiMAX are the promising candidates for the next generation wired and wireless broadband access networks, respectively. However, developing each of them independently suffers from some drawbacks, e.g. EPON does not support mobility and WiMAX can't provide massive bandwidth. Therefore, through convergence of the EPON and WiMAX we can support WiMAX with sufficient bandwidth provided by optical networks and bring mobility features to the EPON [22]. Supporting Ethernet as the common standard interface and having a similar structure in REQUEST, POLL and GRANT schemes make the EPON-WiMAX convergence scheme feasible. However, challenges such as different types of service classes and the way in which EPON and WiMAX talk to each other needs to be considered and addressed efficiently in order to provide smooth ETE data transmission from the wireless domain over the optical networks. In this paper, we address the issues related to the technology integrations of the EPON (TDM EPON and WDM EPON) and wireless (Wi-Fi (802.11) hot spots and WiMAX (802.16)) networks. Our work mainly focuses on improving the ETE delay and providing diverse QoS for different service classes from Wi-Fi hot spot domains over WiMAX, TDM EPON and finally WDM EPON. A new scheme is therefore required to support the WiMAX and EPON convergence and provide an intra-ONU scheduling mechanism. To achieve this, we use different scheduling techniques and comparisons have been made between each of them. A novel upstream intra-ONU scheduling algorithm is described in the paragraph VII . The initial simulation experiments demonstrates the strong impact of using an efficient upstream scheduler in terms of reducing the ETE delay and providing the QoS for diverse service classes. The remainder of this paper is prepared as follows. In section II we briefly discuss the related work according to the optical and wireless integration scenario. Section III and section IV cover issues related to the ETE bandwidth negotiations and QoS in converged scenario. The different scheduling techniques have been discussed in section $\mathrm{V}$ follows by the implementations in section VI. Our proposed scheduling algorithm is detailed in section VII and the section VIII concludes the paper.

\section{RELATED WORK}

To date the wide range of studies has been done for the integration of the optical and wireless technologies in order to save the operational CapEx and OpEx costs and provides ubiquitous broadband connections for the next generation broadband access networks. The integration scheme has been studied in three categories: architecture, physical layer and MAC layer. Shen et al. [4] proposed four architectures for the EPON-WiMAX integration scheme: independent architectures, hybrid architectures, unified connectionoriented architectures and microwave-over-fibre architectures. Tang et al. [1] also proposed three architectures for the converged structure: architecture-independent, hybrid architecture and connection-oriented architecture and investigated the QoS performance between three proposed structures. Luo et al. [10] investigated the feasibility of the integration between optical fibre technology and WiMAX by proposing several architectures. Sarkar et al. [9] proposed the hybrid Wireless-Optical Broadband Access Network (WOBAN) to save network deployment costs and considered the optimal placement of the ONU in WOBAN. Z. Jia et al. [11] proposed a single modulator in Millimetre-Wave-Band Radio-over-Fibre (RoF) which provides both wired and wireless services simultaneously for physical layer integration. Yang et al. [7] addressed the MAC related issues such as QoS provisioning scheme and Dynamic Bandwidth Allocation (DBA) in diverse service classes in EPON and WiMAX integrated scenario. Several technical issues were raised in [4] to support the user mobility (handover), QoS, bandwidth allocation and scheduling for optical and wireless converged scenarios. To the best of our knowledge the traditional single-wavelength PON also known as the Time Division Multiplexed PON (TDM PON) has been considered in almost all of the existing papers for the optical and wireless integration scenarios. In existing TDM PON solutions such as Ethernet PON (EPON), Broadband PON (BPON), Gigabit PON (GPON), Generic Framing Procedure PON (GFP PON), etc., the optical carrier (OC) is shared between the number of ONUs by means of a passive splitter. Therefore, according to the optical network power budget and in order to avoid long queuing delay and provide QoS for different service classes the number of the ONUs is limited by the maximum splitting ratio of 16 and 32 at the maximum distance of $20 \mathrm{~km}$ and 10 $\mathrm{km}$ between the splitter and OLT, respectively. In current TDM EPON, $1 \mathrm{~Gb} / \mathrm{s}$ bandwidth is shared between the number of ONUs, which also limits the total number of the users per ONU in order to avoid QoS degradation. The current TDM EPON architecture needs to be upgraded to the WDM EPON to provide higher bandwidth for ongoing demands for more bandwidth-intensive applications and services. The WDM EPON provides higher bandwidth rather than the traditional TDM EPON by supporting multiple wavelengths over the same fibre infrastructure. Moreover, the TDM EPON needs synchronisation between the OLT and ONUs which adds extra cost and complexity to the network. Therefore, the major contributions of this paper are shortened as follows.

- The integration of the hybrid optical (TDM EPON and WDM EPON) with the hybrid wireless (Wi-Fi and WiMAX) is detailed.

- The ETE MAC-related issues such as bandwidth requests, bandwidth allocations as well as the QoS mappings are detailed from the Wi-Fi hot spots over the WiMAX, TDM EPON and WDM EPON.

- In order to improve the ETE delay and provide QoS for various service classes, different upstream scheduling techniques are considered and comparison 


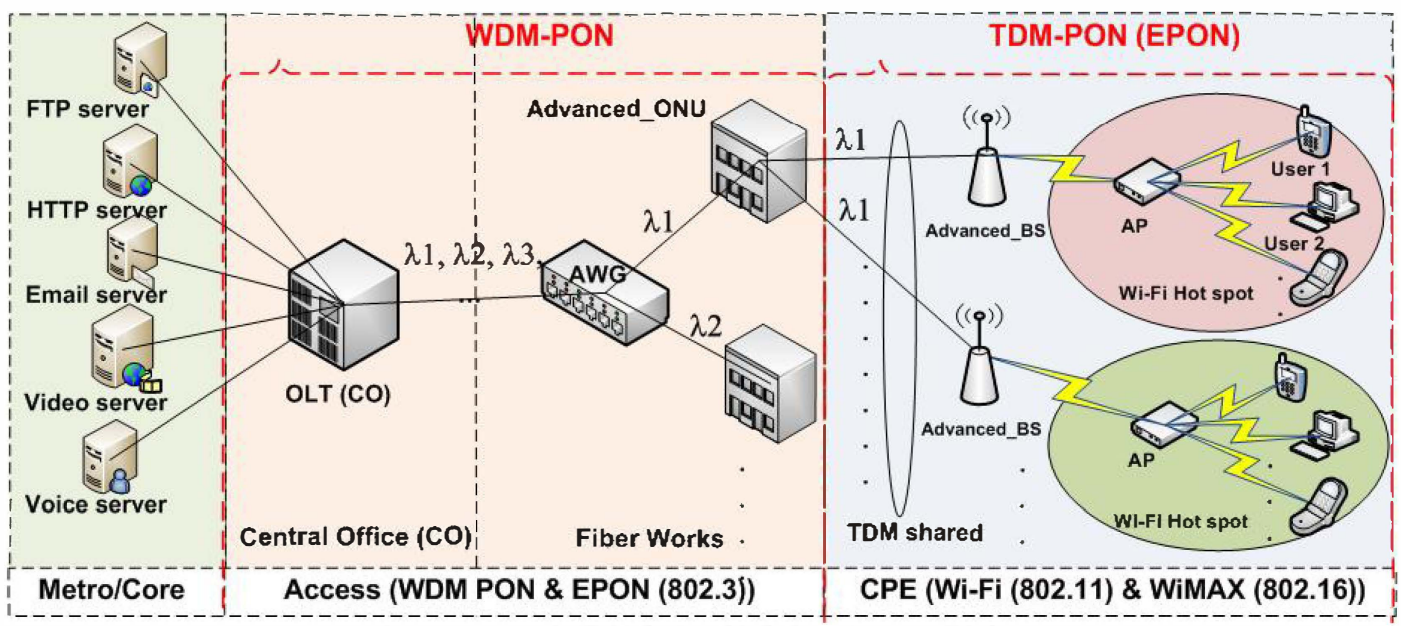

Fig. 1. The Hybrid Optical and Wireless Integrated Scheme

are made between them.

- A novel dynamic upstream scheduling algorithm is also proposed.

\section{BANDWIDTH NEGOTIATIONS}

In this section we discuss how the bandwidth is negotiated over the hybrid optical and wireless converged scenario in three sections: OLT - ONU, ONU - BS and BS - AP Wireless LAN workstations (WLANs).

\section{A. OLT and $O N U$}

The traditional EPON (TDM EPON) is based on a single channel in which the fibre infrastructure carries a single downstream and a single upstream wavelength. The single upstream and downstream channels are typically separated by the Coarse Wavelength Division Multiplexing (CWDM). In TDM EPON, only one type of transceiver is used which results in simple and cost-aware network structure and maintenance. The Multi Point Control Protocol (MPCP) (IEEE 802.3ah) supports bandwidth negotiations between the OLT and the ONUs in traditional EPON [16]. The MPCP is a frame-based protocol includes five 64-byte MAC control messages as follow. REGISTER_REQUEST, REGISTER and REGISTER_ACK which are used in auto-discovery process for the newly joined ONUs. The GATE and REPORT messages are employed to support the upstream bandwidth request and allocation between the OLT and ONUs. In MPCP, bandwidth is allocated by the OLT to the given ONU in the format of the time slots which limits the number of the ONUs to avoid increasing the cycle time linearly. It also adds extra complexity as it needs synchronization between the OLT and ONU. Given the increasing number of bandwidth intensive applications such as: interactive games, Internet Protocol Television (IPTV), video communications, etc., as well as the increasing number of subscribers, the current TDM EPON needs to be upgraded in order to satisfy the ongoing demands in future. Upgrading the single channel TDM EPON to the multi channel WDM EPON is a solution to the traditional TDM EPON shortages. The WDM EPON can support more ONUs and provide higher bandwidth rather than the traditional TDM EPON. The Multi Point Control Protocol Extension (MPCP Extension) is proposed in [19] which can supply wavelength assignment features inside the conventional MPCP. The MPCP Extension enables the OLT to schedule transmission to and reception from the ONUs on any wavelength channel supported by the OLT and the correspondent ONU [19]. We considered WDM EPON at the top level between the OLT and ONU in optical and wireless integration, "Fig. 1". The WDM EPON does not impose any particular structure for the ONU which allows flexible changes on the conventional TDM EPON without additional replacement cost.

\section{B. $O N U$ and $B S$}

In ONU's point of view the network structure from the ONU down to the BS and then wireless workstations in Wi-Fi hot spot domains acts as the TDM EPON. The ONU uses the MPCP in order to share the allocated wavelength from the OLT to its associated BSs. The bandwidth requests from a given BS are received in the format of the MPCP REPORT message on the ONU, which is then replies back using the GATE message to the relevant BS.

\section{BS, APs and WLAN workstations}

In IEEE 802.16 the Subscriber Station (SS) can use two alternatives to request bandwidth from the BS: contention method and polling method. In contention method each SS needs to fight against other SSs to send its bandwidth request in predefined contention window in IEEE 802.16 TDD frame structure which may result in collision. The collided bandwidth requests result in increasing the packet delay. On the other hand, in polling technique, which is the contentionfree method, the BS polls each SS in an active manner during which each SS can send its bandwidth request. Therefore, polling method does not need contention window, which increases the channel utilization, eliminates the collision and significantly reduces the packet delay. In our developed scenario each WLAN workstation needs to fight against other workstations in order to send the bandwidth request to the associated AP. Then, the BS uses the polling method in which APs are actively polled in order to get their bandwidth requests. The APs bandwidth requests will be aggregated and sent towards the ONU inside the MPCP REPORT messages using the scheduler, which is placed inside the BS. The BS 


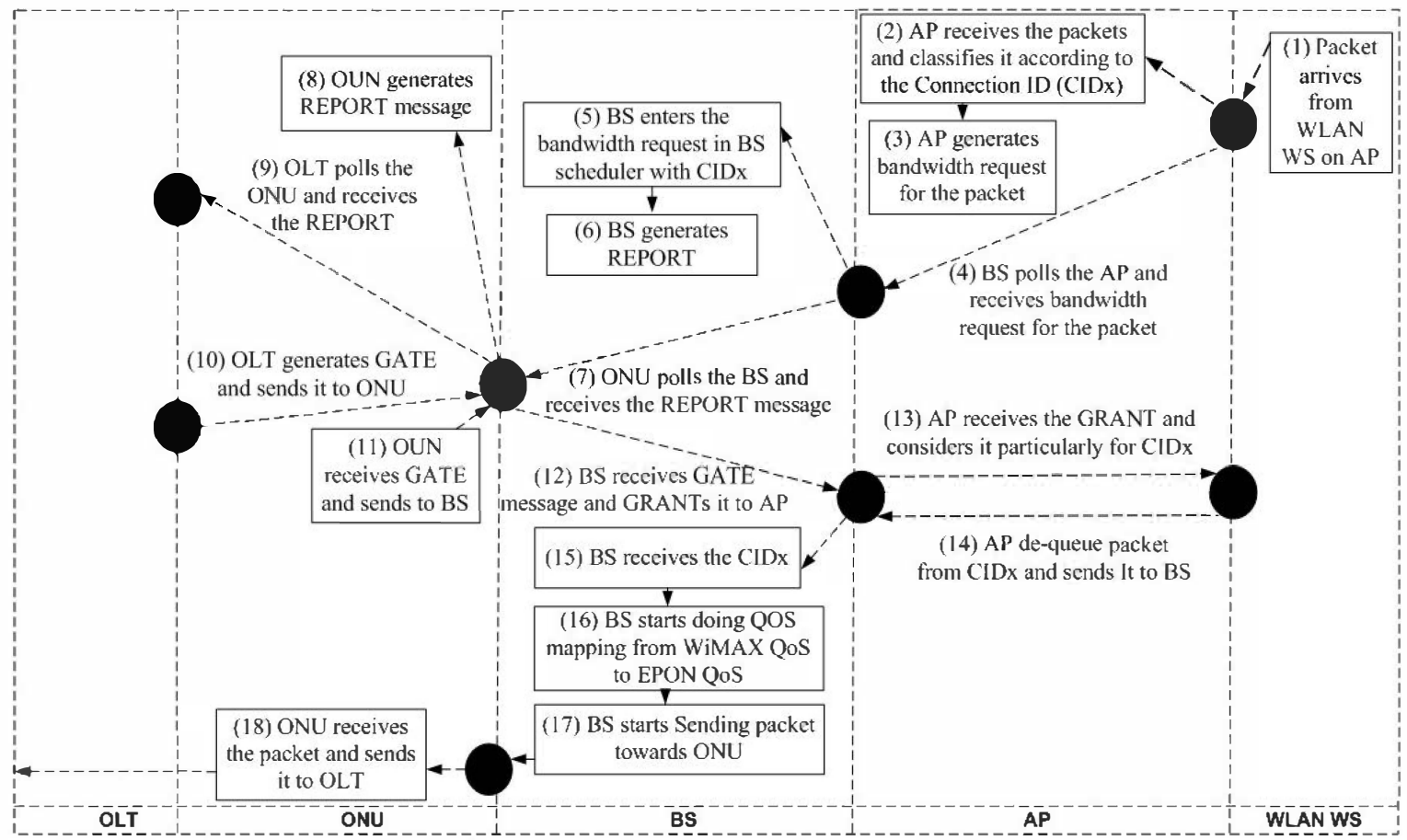

Fig. 2. The MAC Data Exchange between the Hybrid Optical and Wireless Integration

REPORT message includes the total bandwidth required to satisfy traffic generated by all its associated APs. The ONU grants bandwidth to the BS in format of the MPCP GATE message which will be delivered to the AP using 802.16 GRANT message. The scheduler at the AP, which is the lowest level scheduler, then decides how to allocate the granted bandwidth from the associated BS between its connected WLAN workstations. "Fig. 2" depicts the ETE data transmission over the wireless and optical integration scenario.

\section{ETE QOS SUPPORT}

\section{A. Wi-Fi hotspot domain and WiMAX}

The lack of the built-in QoS mechanisms in 802.11 makes it very difficult to provide sufficient ETE QoS for different service classes specifically for the delay-sensitive applications and services such as VoIP, interactive games, streaming multimedia, etc. The $802.11 \mathrm{e}$ is being proposed as the upcoming standard for the IEEE 802.11 enhancement of service definitions. The wide range of 802.11 QoS mechanisms has been proposed in the literature which can be classified as: link adaptation in the physical layer, service differentiations in the MAC layer, bandwidth reservation and admission control in the MAC layer and higher layers. The service differentiation in the MAC layer is achieved by the two main methods: priority and fair scheduling [21]. While the former prioritises the channel access to different priority queues, the latter divides channel access fairly among different service classes according to the given weight. The Enhanced Distributed Coordination Function (EDCF) [8], Persistent Factor DCF (P-DCF) [13], Distributed Weighted Fair Queue (DWFQ) [14, 15] and the Distributed Fair Scheduling (DFS) $[14,17]$ are some of the service class differentiation mechanisms in 802.11. In our implementation we consider QoS provisioning scheme by means of priority queuing in which service classes in Wi-Fi hot spots are prioritised into four priority queues as: Expedited Forwarding (EF), Assured Forwarding (AF), Excellent Effort (EE) and Best Effort (BE) services. Two medium access protocols have been developed in 802.11: Distributed Coordination Function (DCF) and Point Coordination Function (PCF). Each wireless sub-frame includes a contention period for the DCF and a contention free period for the optional PCF. During the contention free period, each Access Point (AP) polls workstations with high priority service classes like the EF service class periodically while other workstations with lower service classes should fight against each other to get the wireless channel during the contention period.

\section{B. WiMAX and EPON}

WiMAX and EPON both use priority queuing to provide the ETE QoS for their correspondent subscribers; however the QoS scheme is different in each of them. It is based on Differentiated Service (DifServ) in EPONs with three defined Classes of Services (CoS) [12] [4] as: Expedited Forwarding (EF), Assured Forwarding (AF) and the Best Effort (BE) services. EPON can support up to eight different priority queues. The QoS is based on Integrated Service (IntServ) in WiMAX with five defined $\mathrm{CoS}$ as: Unsolicited Grant Service (UGS), Real-Time Polling Service (rtPS), Extended RealTime Polling Service (ertPS), non-Real-Time Polling Service (nrtPS) and the Best Effort service (BE) [10]. In our implementation in order to define how to support the QoS in optical and wireless integrated scheme, we mapped four service classes of $\mathrm{Wi}-\mathrm{Fi}$ to four correspondent WiMAX service classes. Therefore, the EF, AF, EE and the BE service classes in Wi-Fi treats as the UGS, rtPS, nrtPS and the BE service types, respectively in WiMAX. Similar to the work in [7], we have also mapped the five WiMAX service types of UGS, ertPS/rtPS and the nrtPS/BE into three service types of 
UGS, rtPS and the BE, respectively. It is done because of the closed similarities between the ertPS and rtPS and between the nrtPS and BE services. Therefore, the ONU treats the UGS, ertPS/rtPS and the nrtPS/BE service types in WiMAX as $\mathrm{EF}, \mathrm{AF}$ and $\mathrm{BE}$ service types, respectively in EPON. Generally speaking, four service classes of EF, AF and $\mathrm{EE} / \mathrm{BE}$ in Wi-Fi domain will be finally mapped to the three service classes of EF, AF and BE in EPON, respectively after going through the entire integrated domain. This means the EE service classes from the Wi-Fi will be treated as the BE service class at the end.

\section{SCHEDULING TECHNIQUES}

In this paper, the ONU communicates directly with each BS so the BS is also known as the wireless gateway as it is a gateway between the wireless and optical worlds. We considered that each ONU connects to $16 \mathrm{BSs}$ so, the maximum available bandwidth for each $\mathrm{BS}$ is equal to the total upstream available bandwidth, which is $1 \mathrm{Gbps}$, divided by the total number of BSs: 1000Mbps $(1 \mathrm{G}) / 16=62.5 \mathrm{Mbps}$. Therefore, the link between each BS and the ONU is a potential bottleneck. The ONU grants bandwidth to each BS by means of TDM EPON in format of the GATE messages during which each BS gets control of the up-link shared bandwidth for a small fraction of the time in cyclic fashion. The BS needs to queue its subscriber data between two consecutive time slots of different priority queues based on the diverse traffic classes. When the allocated bandwidth from the ONU received on BS, the BS scheduler should consider in which order the data from different priority queues should be sent towards the up-link fibre. Letting all the priority queues to send data at the same time will result in collusion. In this paper, we have applied six specific scheduling techniques for the integrated scenario in order to provide the ETE QoS for different service classes as follows. The Priority Queuing (PQ), Custom Queuing (CQ), Custom Queuing with Low Latency Queue (CQ-LLQ), Weight Fair Queuing (WFQ), Weight Fair Queuing with Low Latency Queue (WFQLLQ) and the Modified Deficit Round Robin (MDRR). We have also proposed a novel scheduling algorithm for the integrated scenario in order to make use of the excessive amount of the bandwidth. In this paper, in all the scheduling techniques, the traffic is first queued based on its priority in the correspondent priority queue. The priority is assigned when the traffic is generated by the source node. However, when the allocated time slot from the ONU arrives the way in which the scheduler treats each priority queue is different between six techniques. For simplicity, three priority queues which are representing three CoSs have been considered in this paper. The traffic from the highest $\mathrm{CoS}$ to the lowest CoS is queued from the highest to the lowest priority queues, respectively. In PQ technique, a queue which has assigned to the first priority is always served as long as it is not empty. The second priority queue is served if the first priority queue is empty. And finally, the third priority queue, which carries the lowest $\mathrm{CoS}$, is served if the first and the second priority queues are empty. In CQ technique, queue with highest priority gets the highest byte counts and the traffic is sent from the highest priority to the lowest priority based on their assigned byte counts in round robin fashion. In CQ-LLQ technique, the low latency queue gets the highest priority and will be served first regardless of its actual priority and its byte counts. Then the other priority queues will be served in round robin fashion based on CQ technique when the low latency queue gets empty. In WFQ technique, each queue is prioritised based on an aggregated weight in which queue with the higher weight gets the better services. In WFQ-LLQ technique, queue with the low latency attribute serves as the highest priority queue regardless of its actual weight. The other queues will be served with regard to the WFQ policy if the low latency queue is empty. In MDRR technique, the allocated time slot from the ONU is split among the different priority queues so each priority queue gets the percentage of it with regard to its assigned priority in a round robin fashion.

\section{IMPLEMENTATIONS AND RESULTS}

We validated the hybrid optical and wireless integration architecture in a typical converged scenario using OPNET Modeler [18] based on the parameters defined in Table I. In converged scenario we assigned a single wavelength to a typical ONU statically by means of WDM EPON. The allocated wavelength to the ONU is shared between 16 BS by means of TDM EPON in which each BS takes control of the ONU's single wavelength for a short time in a cyclic fashion. The Interleaved Polling with an Adaptive Cyclic Time (IPACT) [23] is used inside the ONU in order to allocate time slot to the BSs. The IPACT is placed inside the ONU and polls the BSs in a round-robin fashion then grants time slot dynamically to each with regard to the reported queue length. The Wi-Fi traffic can be transferred towards the OLT only during the allocated time slot. The EPON cycle time in which all $16 \mathrm{BS}$ will be served is set to $2 \mathrm{~ms}$ and the guard time between different cycles is set to $1 \mu \mathrm{s}$ to avoid data overlapping between the various transmission cycles. We selected the voice over IP (PCM quality), streaming video and the FTP as three Types of Services (ToS) with UGS, rtPS and $\mathrm{BE}$ scheduling types, respectively. The Maximum sustained traffic rate are set to $96 \mathrm{Kbps}, 0.5 \mathrm{Mbps}$ and $0.5 \mathrm{Mbps}$ and the Minimum reserved traffic are set to $96 \mathrm{Kbps}, 384 \mathrm{Kbps}$ and $64 \mathrm{Kbps}$ with 10, 10 and 30 milliseconds maximum latency for the UGS, rtPS and the BE service classes, respectively per workstation. The scheduler is the most important module inside the BS which arbitrates access to the upstream shared fibre between different priority queues. It is the only component that receives the allocated time slot from the ONU in the format of the GATE message and then shares it between queues with different QoS requirements. The start time and the length of the time during which the BS scheduler has access to the upstream shared bandwidth are addressed in GATE message. We considered five different priority queues inside each BS based on WiMAX's five diverse classes of services. The WiMAX priority queues are then mapped into the three priority queues with regard to the mapping procedure explained in section IV. Therefore, the BS scheduler interacts with three priority queues: a queue for the UGS service which has the same priority as EF service in EPON, a queue for the join rtPS service (ertPS and rtPS) which has the same priority as AF service in EPON and a queue for the join BE service (nrtPS and BE) which has the same priority as BE service in EPON. The scheduler inside 


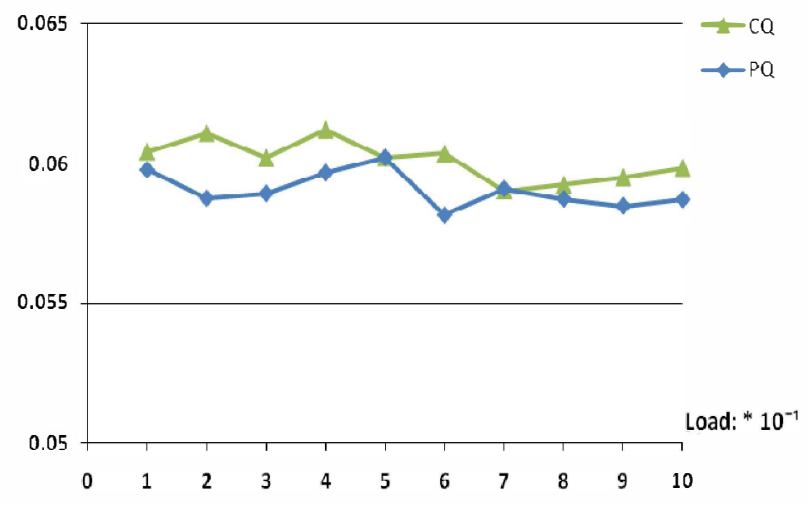

Fig. 3. UGS average ETE delay over the CQ and PQ scheduling

BS allows traffic transmission only after receiving a GATE message from the ONU. The first GATE message arrives when the BS finishes its registration process. After that, the BS needs to send a REPORT message at the end of the each time slot to inform the ONU about the size of the data buffered in each priority queues and then waits for the next GATE message to arrive before sending any traffic. The way in which the scheduler reads data from different priority queues after receiving the allocated time slot from ONU is a controversial issue which needs to be addressed efficiently in order to provide the ETE QoS for diverse service classes. To achieve this, we have provided six different scheduling techniques which are distributed in two levels (AP and BS) as follow. The Priority Queuing (PQ), Custom Queuing (CQ), Custom Queuing with Low Latency Queue (CQ LLQ), Weight Fair Queuing (WFQ), Weight Fair Queuing with Low Latency Queue (WFQ_LLQ) and the Modified Deficit Round Robin (MDRR). Three types of queues by three levels of priorities are provided for the UGS, rtPS and the BE service classes. We run the simulation for 1200 seconds with a seed of 128 and 100 values per statistic to investigate how employing different scheduling techniques would affect the ETE QoS for the WLAN subscribers from the Wi-Fi domain over the optical infrastructure. In PQ scheduling mechanism queues are prioritised based on their correspondent priority index. In this technique lower priority queues send packets only if the higher priory queues are empty, e.g. in our scenario the UGS priority queue sends packets as long as it is not empty, the rtPS priority queue sends packet if the UGS queue is empty and the BE sends packet if the UGS and the rtPS queues are empty. The CQ scheduling technique serves three different priority queues in round robin fashion based on the correspondent byte counts which are set to 14000,8000 and 2000 for the UGS, rtPS and the BE priority queues, respectively in our converged scenario. In MDRR scheduling technique each priority queue sends its buffer data only during its correspondent percentage of the allocated time slot. We considered $50 \%, 30 \%$ and $20 \%$ of the allocated time slot for UGS, rtPS and BE priority queues, respectively, e.g. the UGS priority queue sends its buffered data only in the first $50 \%$ of the arrived time slot while the rtPS and BE queues send their data in remained $30 \%$ and $20 \%$ in sequence right after the UGS queue. As "Fig. 3" reveals the average ETE delay for the UGS service class is significantly reduced by the

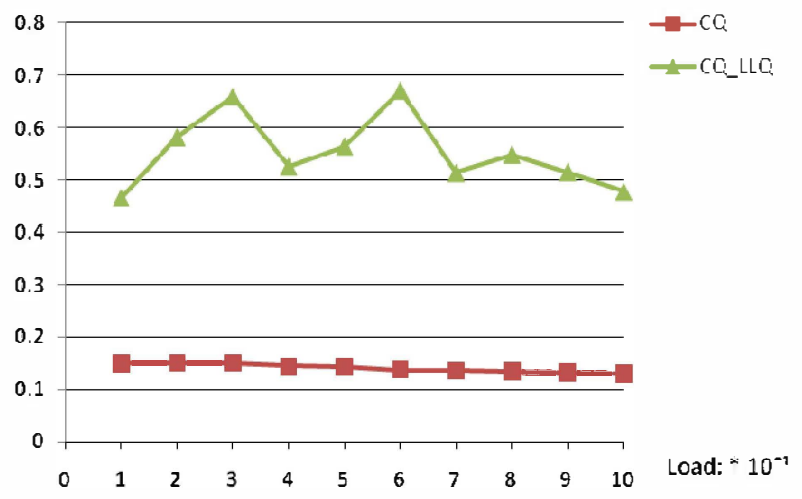

Fig. 4. rtPS average ETE delay over the CQ and CQ_LLQ scheduling

ratio of 1.02 when the $\mathrm{PQ}$ is applied in comparison to the CQ scheduling technique. This is because the PQ scheduler puts strict priority on the highest priority queue (UGS) in which the other lower priority queues (rtPS and BE) will send packets if the UGS priority queue is empty. The CQ treats three priority queues in a round robin fashion each with correspondent byte counts which means longer delay for the UGS and shorter delay for the rtPS and BE in comparison with the PQ mechanism. In CQ LLQ technique, a queue which gets the attribute of the low latency, receives the highest priority and it sends packets as long as it is not empty regardless of the associated byte counts. Other queues are served based on CQ technique if the low latency queue is empty. To show how this technique can affect the ETE delay for different priority queues, we set the BE priority queue with LLQ attribute in which the UGS and the rtPS are served according to their byte counts if the LLQ queue is empty. We then investigate the impact of the CQ_LLQ scheduling technique on rtPS ETE delay. As "Fig. 4" depicts, the CQ_LLQ increases the rtPS average ETE delay by the ratio of 3.93 in comparison with the CQ scheduling technique. That is because in the CQ which is byte count-based technique, the rtPS priority queue sends packets right after the 14000 bytes sent from the UGS in a round robin fashion. However, in the CQ_LLQ with regard to the LLQ attribute of the BE, the rtPS should wait for the BE queue to get empty and UGS queue to send 14000 bytes and then start sending 8000 bytes which repeats continuously until the end of the received time slot. Therefore, the rtPS priority queue will suffer the longer average ETE delay in CQ_LLQ rather than the CQ technique. In WFQ mechanism queues with higher priority get the higher weight and send packets according to their assigned weights. We considered 60,30 and 1.0 as the correspondent weights to UGS, rtPS and the BE priority queues, respectively. In WFQ_LLQ, queue with low latency attribute sends the packets if it is not empty regardless of its correspondent weight. Other queues will be treated according to their weights. We assigned the LLQ attribute to the BE priority queue in which its allocated weight will be ignored during the service time. Other queues will be treated according to their weights. The UGS and rtPS queues will be treated regarding their weights if the LLQ is empty. "Fig. 5" depicts the impact of the WFQ LLQ scheduling in comparison with the CQ and CQ_LLQ techniques on the average ETE delay for BE 
priority queue. As it reveals, the WFQ_LLQ decreases the average ETE delay for BE priority queue by the ratio of 1.350 and 1.296 in comparison with the CQ and the CQ_LLQ scheduling techniques, respectively. It also revealed that employing the CQ_LLQ helps decreasing the average ETE delay in $\mathrm{BE}$ queue by the ratio of 1.041 in comparison with the CQ technique. "Fig. 6" depicts the impact of the CQ, the CQ LLQ and the PQ scheduling techniques on the average ETE delay for the UGS priority queue. As it reveals, the CQ LLQ has the strongest negative impact on the average ETE delay for the UGS service class in comparison with the $\mathrm{CQ}$ and the PQ scheduling techniques. The CQ LLQ increases the average ETE delay for UGS service class by the ratio of 1.430 and 1.455 when it is compared with the CQ and $P Q$, respectively. That is because in CQ_LLQ according to the low latency attribute of the BE priority queue, the UGS queue sends packet if the BE queue is empty while in CQ and PQ scheduling the UGS is the first priority queue which is served by the scheduler. As it is depicted in "Fig. 7", the MDRR scheduling technique helps improve the average ETE delay for the rtPS priority queue when the network load increases linearly by granting the different portions of the allocated time slot received from the ONU strictly to the three correspondent queues.

TABLE I. SIMULATION PARAMETERS

\begin{tabular}{|l|l|}
\hline \multicolumn{2}{|c|}{ TDM EPON \& WDM EPON } \\
\hline Speed: ONU - AWG, AWG - OLT, OLT - app. server & $1 \mathrm{Gbps}$ \\
\hline Number of application server in CO & 3, Quad-play \\
\hline EPON Cycle Time and Guard time & $2 \mathrm{~ms}, 1 \mu \mathrm{s}$ \\
\hline Distance between (OLT, AWG) \& (AWG, ONU) & $20 \mathrm{~km}, 5 \mathrm{~km}$ \\
\hline \multicolumn{2}{|c|}{ WiMAX and Wi-Fi } \\
\hline Total number of BS per ONU and AP per BS & 16,1 \\
\hline Number of WLAN workstations per AP & 8 \\
\hline WiMAX Channel Capacity & $20 \mathrm{MHZ}$ \\
\hline WiMAX Duplexing and Frame Duration & TDD, $5 \mathrm{~ms}$ \\
\hline Uplink and Downlink modulation and coding & QPSK $1 / 2$ \\
\hline
\end{tabular}

\section{PROPOSED SCHEDULING Algorithm}

We propose a dynamic scheduling algorithm which operates over the BS. This scheduling algorithm is the only module that defines which priority queue, for how long and in which order access the up-stream shared fibre. The proposed scheduler starts working inside BS immediately after a sample $\mathrm{BS}$, named as $B S_{i}$ finishes its registration process using autodiscovery messaging format in IEEE 802.3ah. The $B S_{i}$ sends the bandwidth request using a REPORT message in IEEE 802.3ah. The scheduler has full access to all the priority queues which provided for diverse traffic types inside the $B S_{i}$. The parameters inside the scheduling algorithm for a sample $B S_{i}$ are defined in Table II. The scheduler starts working immediately after receiving the allocated time slot from ONU.

\section{TABLE II. PARAMETERS FOR SCHEDULING ALGORITHM}

\begin{tabular}{|l|l|}
\hline UGS, rtPS, BE & Three priority queues defined for scheduler \\
\hline$\alpha, \mathrm{B}, \delta$ & Guaranteed bandwidth for UGS, rtPS and BE (\%) \\
\hline$B C_{i}^{c}$ & Byte Counts for queue $i$ \\
\hline$Q_{i}^{\text {length }}, B_{i}^{\text {grt }}$ & Queue length of queue $i$, Bandwidth granted to queue $i$ \\
\hline$B_{\text {total }}^{\text {excess }}$ & Total extra bandwidth collected from all queues \\
\hline$B_{i}^{\text {excess }}$ & Extra bandwidth granted to queue $i$ \\
\hline
\end{tabular}

For simplicity, we have considered the three priority queues inside the $B S_{\mathrm{j}}$ as: UGS for the first priority queue, rtPS for the second priority queue and BE for the third priority queue. Lets $\alpha, B$ and $\delta$ be the guaranteed bandwidth for the UGS, rtPS and the BE priority queues, respectively which means that the scheduler should always guarantee that these three priority queues can send their buffered data in $\alpha, B$ and $\delta$ percentages of the received time slot. The $\alpha, B$ and $\delta$ values are based on SLA specifications to guarantee the ETE QoS of diverse service classes and can vary between different BSs. The guaranteed bandwidth for three service classes can convert to byte counts using (1) where $B C_{i}^{c}$ is the byte counts for queue $i=1 \ldots 3, K_{i}^{c}$ is the guaranteed bandwidth for queue $i=1 \ldots 3$ and MTU is the Maximum Transmission Unit, which is set to 1500 bytes in our implementations.

$$
B C_{i}^{c}=\left(K_{i}^{c * 512)+\mathrm{MTU}}\right.
$$

Let $Q_{i}^{\text {length }}$ be the queue length for $i=1,2,3$ queue when the scheduler sends REPORT message towards the ONU. On the arrival of the time slot, the scheduler checks this value against the guaranteed byte counts for each priority queue which can have three cases as follows. In case one (2), the queue length reported to the $\mathrm{ONU}$ is less than the guaranteed byte counts. In this case the scheduler grants the byte counts equal to the queue length to the corresponding queue (3) and saves the extra byte counts (4).

$$
\begin{aligned}
& Q_{i}^{\text {length }}<B C_{i}^{c}, \text { for } i=1,2,3 \\
& B_{i}^{\text {grt }}=Q_{i}^{\text {length }} \\
& B_{\text {total }}^{\text {excess }}=B C_{i}^{c}-Q_{i}^{\text {length }}
\end{aligned}
$$

In case two (5) the queue length reported to the ONU is equal to the guaranteed byte counts. In this case the scheduler grants the exact guaranteed byte counts to the corresponding queue (6) and there is no byte counts to save.

$$
\begin{array}{ll}
Q_{i}^{\text {length }} & =B C_{i}^{c}, \text { for } i=1,2,3 \\
B_{i}^{\text {grt }} & =B C_{i}^{c}
\end{array}
$$

In case there (7), the queue length reported to the ONU is more than the guaranteed byte counts. In this case, the scheduler grants the guaranteed byte counts plus the portion of the excessive bandwidth collected in (4) to the overloaded priority queues (8).

$$
\begin{aligned}
& Q_{i}^{\text {length }}>B C_{i}^{c}, \quad \text { for } i=1,2,3 \\
& B_{i}^{\text {grt }}=B C_{i}^{c}+K_{i}^{c} * B_{\text {total }}^{\text {excess }}
\end{aligned}
$$

\section{CONCLUSION AND WORK IN PROGRESS}

The optical and wireless technology integrations have been considered as a promising candidate for the next generation broadband access networks for quite some time. The integrated scenario takes into account the massive bandwidth of optical networks as well as the mobility features of the wireless domains for subscriber stations. It is also an economic solution for the rural area where the T-1/E-1, CM, 


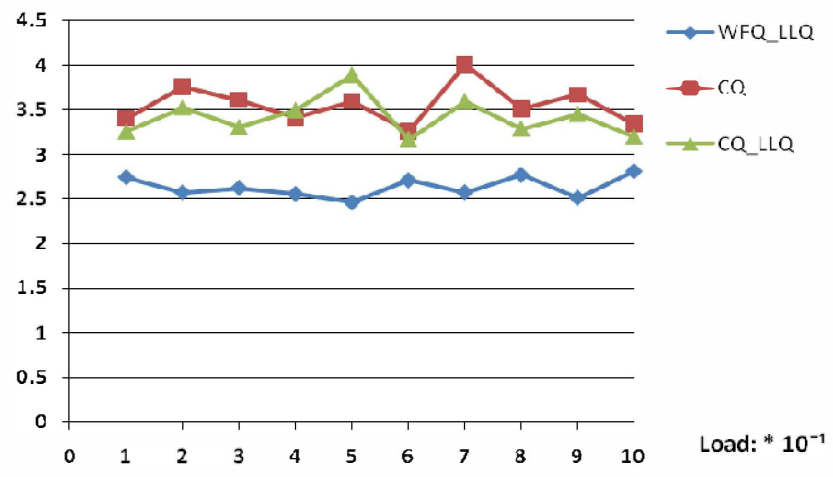

Fig. 5. BE average ETE delay (sec) over different scheduling techniques

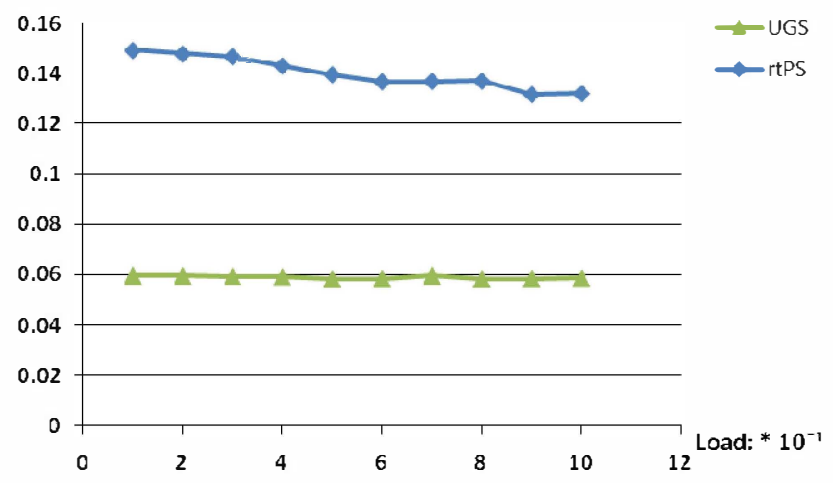

Fig. 7. UGS and rtPS average ETE delay (sec) over MDRR scheduling

DSL or even optical infrastructures are either unreachable or too costly. The current TDM EPON which is considered in almost all the previous works need to upgrade to the WDM EPON to provide higher bandwidth for ongoing demands for more bandwidth-intensive applications and services. The WDM EPON provides excellent scalability as it supports multi-wavelengths over the same fibre infrastructure. In this paper, we proposed a novel up-link scheduling algorithm for the hybrid optical and wireless technology integrations. We have also compared different up-stream scheduling techniques which show the strong effect on providing ETE QoS for diverse CoS during the OPNET simulations. Our current work is to further implement and evaluate of the proposed up-link scheduler algorithm with the purpose of improving up-stream bandwidth utilization with regard to the actual need of each priority queue which changes by network traffic pattern. During the next phase of our research we will be conducting the detailed implementation of our new inter-ONU scheduling which will provide the dynamic bandwidth allocation between different priority queues.

\section{ACKNOWLEDGEMENTS}

The authors would like to acknowledge the support of University of Ulster for providing VCRS scholarship and IUATC for funding the joint work with BT.

\section{REFERENCES}

[1] T. Tang et al., "Performance Analysis of Bandwidth Allocation of Convergence of WiMAX and EPON", IEEE CS, 2009, 662-665.

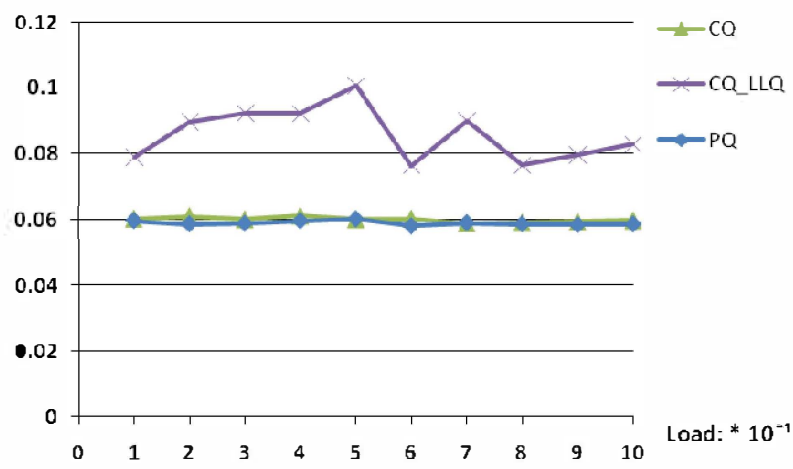

1 Fig. 6. UGS average ETE delay (sec) over different scheduling techniques

[2] Y.Luo et al., "Resource Management for Broadband Access over TimeDivision Multiplexed Passive Optical Networks", Sept.-Oct. 2007, 21:5, 20-27.

[3] ITU-T G.984.x, available at: http://www.itu.int/rec/T-REC-G/e

[4] G. Shen et al., "FMC Architectures for Broadband Access: Integration of EPON and WiMAX", IEEE Com. Mag., 45:8, Aug 2007, 44 - 50.

[5] IEEE 802.3ah, available at: http://www.ieee802.org/3/efm.

[6] B. Skubic et al., "A Comparison of Dynamic Bandwidth Allocation for EPON, GPON, and Next-Generation TDM PON", IEEE Com. Mag. Mar. 2009, 47:3, 40-48.

[7] K. Yang et al., "Convergence of Ethernet PON and IEEE 802.16 Broadband Access Networks and its QoS-Aware Dynamic Bandwidth Allocation Scheme", IEEE JSA, Feb. 2009, 27:2,101-115

[8] IEEE Std 802.11e-D3.3, "Part 11: Wireless LAN Medium Access Control (MAC) and Physical Layer (PHY)", Oct. 2002.

[9] S. Sarkar et al., "Hybrid wireless-optical broadband- access network (WOBAN): A review of relevant challenges", JLT, Nov. 2007, 25: 11, 3329-3340.

[10] Y.Luo et al., "Integrating Optical and Wireless Services in the Access Network", OSA, 2006.

[11] Z. Jia et al., "Simultaneous generation of independent wired and wireless services using a single modulator in millimetre-wave-band radio-over-fibre systems", IEEE PTL., Oct. 2007, 19: 20, 1691-1693.

[12] C. Assi et al., "Dynamic bandwidth allocation for quality-of-service over Ethernet PONs", IEEE JSA, Nov. 2003, vol. 21, 1467-1477.

[13] Y. Ge et al., "An Analytical Model for Service Differentiation in IEEE 802.11", IEEE ICC, May 2003, vol. 2, 1157-62.

[14] A. Banchs et al., "Providing Throughput Guarantees in IEEE 802.11 Wireless LAN", IEEE WCNC, 2002, vol. 1, 130-38.

[15] A. Banchs et al., "Distributed Weighted Fair Queuing in 802.11 Wireless LAN", IEEE ICC, 2002, vol. 5, 3121-27.

[16] N. Moradpoor et al., "Simulation and Performance Evaluation of Bandwidth Allocation Algorithms for EPONs", OPNETWORK2010.

[17] N. Vaidya et al., "Distributed Fair Scheduling in a Wireless LAN," ACM MOBICOM, 2000, 167-78.

[18] OPNET Modeler 16.0, available at: www.opnet.com.

[19] M. McGarry et al., "WDM Ethernet Passive Optical Networks", IEEE Com. Mag., 2006, 44: 2

[20] J. Zhang et al., "Next-Generation PONs: A Performance Investigation of Candidate Architectures for Next-Generation Access Stage 1," IEEE Com. Mag., Aug. 2009, 47: 8, 49-57.

[21] W. Pattara-Atikom et al., "Distributed Mechanisms for Quality of Service in Wireless LANs", IEEE Wireless Com., June 2003, 26-34.

[22] S.Ou et al., "Integrated DBA in Converged Passive Optical Netwroks and IEEE 802.16 Netwroks", IEEE System Journal, Dec.2010, 4:4.

[23] G. Kramer et al., "IPACT a dynamic protocol for an EPON", IEEE Com. Mag., Feb. 2002, vol. 40, 74-80.

[24] D. Remondo et al., "Integration of Optical and Wireless Technologies in the Metro-Access: QoS Support and Mobility Aspects", NGI, 2009. 